\title{
Der zweite Papyrusfund von Kahun und die zeitliche Festlegung des mittleren Reiches der ägyptischen Geschichte.
}

\author{
Von Ludwig Borchardt.
}

Im Anfang des Jahres 1899 tauchten in Kairo einige Fragmente von MittlerenReichs-Papyri auf, deren Herkunft nicht zu verkennen war; die auf ihnen vorkommenden Königs- und Ortsnamen wiesen deutlich auf die von Petrie entdeckte Stadtruine der 12. Dynastie bei Illahun, das sogenannte Kahun. Die zuständigen Beamten des Kairiner Museums wurden davon in Kenntnifs gesetzt und gleichzeitig auch dem gerade in Ehnasje anwesenden Dr. ScHäFEr davon Mittheilung gemacht. Diesem gelang es, eine gröfsere Anzahl der Fragmente zu erwerben. Gleichzeitig brachte auch Grenfell davon nach Kairo. Den bei Weitem grö̊sten Theil des Fundes aber war Dr. Reinharot so glücklich ankaufen zu können, und er befindet sich heute, dank seiner Güte, im Königl. Museum zu Berlin. Um die Herkunft dieses Schatzes sicherzustellen, erbat sich das Berliner Museum dann noch die Erlaubnifs, Nachforschungen an der vermuthlichen Fundstelle vornehmen zu dürfen, was die Ägyptische Alterthümerverwaltung auch in zuvorkommendster Weise gestattete.

Die Untersuchungen an Ort und Stelle fanden in den Tagen vom 12. his 26. Juni d. J. statt und ergaben folgenden Befund. Aufserhalb der alten Stadt liegen drei Schutthügel, die alten Abfuhrplätze vor den Thoren. Der östliche
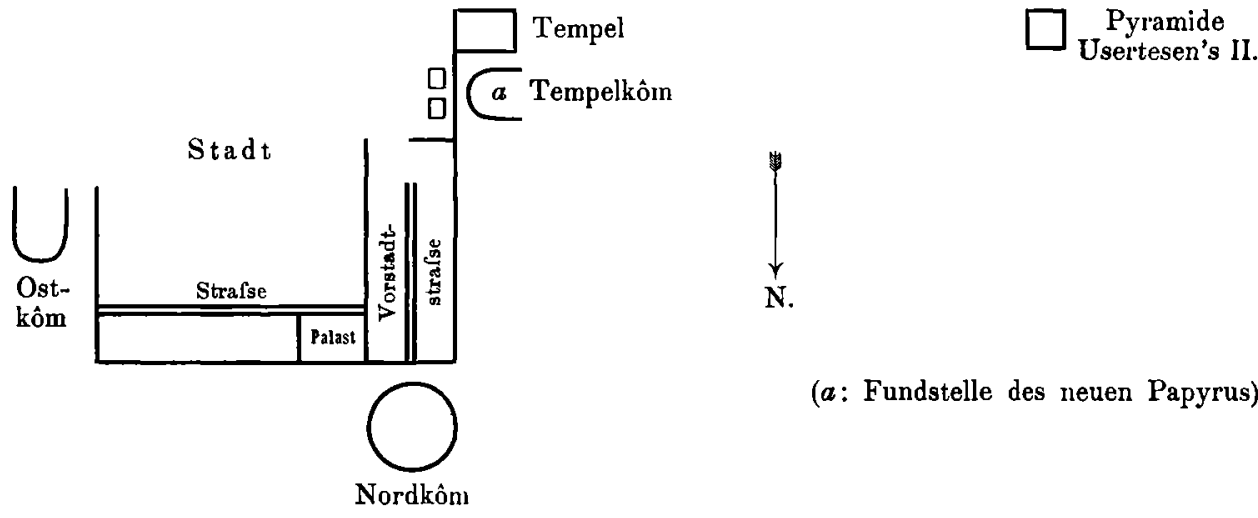

(a: Fundstelle des neuen Papyrus)

und nördliche davon waren - wenigstens an den untersuchten Stellen - ohne Papyri; nur in dem neben dem Tempel gelegenen, den auch die Anwohner als die Fundstelle bezeichneten, waren Papyri nachzuweisen, nicht gerade reichlich, aber genügend, um diesen Platz als den Fundort der angekauften Stücke be- 
zeichnen zu können. Ein dort gefundener Brief war an denselben Tempelvorsteher Kmiw gerichtet, der auch in den gekauften Stücken einige Male auf Briefadressen vorkommt.

Da anzunehmen war, dafs der Tempelkôm sich im Wesentlichen aus dem Müll und den Abgängen des 'Tempels gebildet habe, so lag die Vermuthung nahe, dals die dort gefundenen Papyrusfetzen Theile der Tempelcorrespondenz sein würden. Eine nähere Durchsicht des Fundes bestätigte dies sogleich, und jetzt, wo die ganze Masse in Berlin wenigstens vorläufig verglast ist — auch GRIFFITH stellte die ihm von Grenfel übergebenen Stücke dem Berliner Museum freundlichst leihweise zur Verfügung - , kann man wohl sagen, dafs kein Stück irgend einer Privatcorrespondenz sich in dem Funde befindet; es sind nur Tempelacten und die dazu gehörigen Belege. Eine kurze Übersicht mag dies zeigen.

Ungerechnet die in Kairo aufbewahrten Fragmente, besteht der Fund aus:

41 Tafeln eines Tempeltagebuchs,

5 " Festlisten,

5 "Priesterlisten,

63 " Rechnungen des Tempels,

53 "Briefe an Tempelbeamte,

6 " pappeartige Conglomerate,

26 " vorläufig nicht rubricirte Fragmente,

zusammen 199 Tafeln von im Durchmesser etwa $25 \times 40 \mathrm{~cm}$.

Die Grölse der Stücke variirt sehr, von wenigen Centimetern bis zu der ganz respectablen Länge von $3 / 4 \mathrm{~m}$. Da bisher noch die Zeit mangelte, Versuche zum Zusammensetzen der Fragmente zu machen, so läfst sich noch nicht überblicken, inwiefern die oben gegebenen Zahlen noch modificirt werden müssen.

Die Briefe sind nur an einell selır beschränkten Kreis von Tempelbeamten gerichtet. Am häufigsten $(15 \mathrm{Mal})$ tritt schreiber $H r-m-s i f$ « auf, der übrigens auch einige (3) Male als Absender erscheint. Nächst ihm ist besonders lıäufig (5 Mal) vertreten, dann folgen zwei weitere Priester, $s=$ th $S b k$-nht und $=$ nerkw $s b k-m-s\} f$. Merdig ist ein leider nur in der

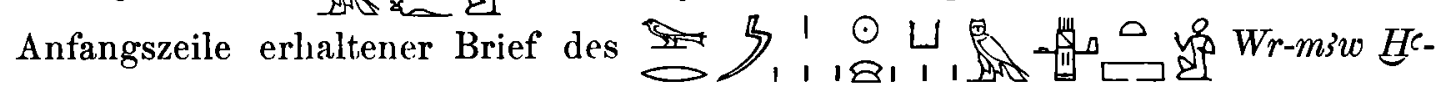
$k ; w-r e-m-c h c t$ an den 'Tempelvorsteher von Illahun. Auch Briefe mit der roth daneben geschriebenen Antwort, wie sie auch GrIFriTH schon publicirt hat, finden sich wieder. Unter den Briefabschriften, die auch in unseren Acten vorkommen, betrifft eine Reihe die fortgesetzten, aber erfolglosen Mahnungen um Lieferung von Opfern. Die Briefe unterscheiden sich in ihrer Fassung sehr von einander, je nach dem Range des Empfängers; neben schwülstigen, langen Briefen befinden sich ganz kurze Bestellzettel. Einen derselben wollen wir hier folgen lassen, da er für die weiteren Ausführungen noch von Interesse sein wird. 


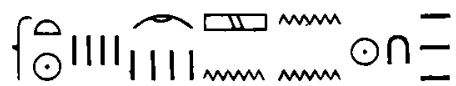

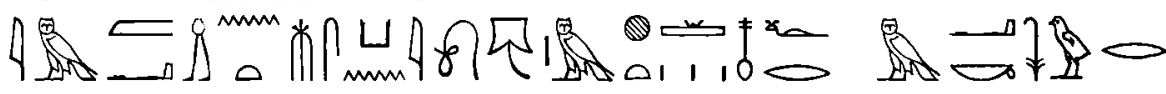

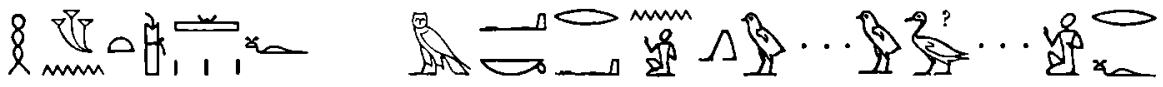

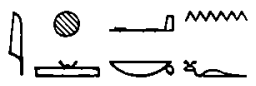

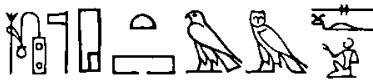

"Jahr 4, 4. Erntemonat, am 13.

"Lafs ein gutes Rindsleder bringen. Ich brauche es(?).

"Ich schicke den NN. danach. Gieb es ihm.

"Der Tempelschreiber $H r-m-s i f$. "

Die Quittungen über gelieferte Opfer beziehen sich auf die Götter:

$$
\begin{array}{ll} 
& \text { "Anubis auf seinem Berge" } \\
& \text { "Suchos von Sdt" }
\end{array}
$$

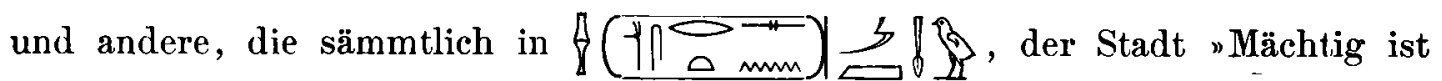
der selige Usertesen ", verehrt wurden. Natürlich kommen auch Opfer für den verstorbenen König Usertesen II. und Angehörige seiner Familie vor, z. B.:

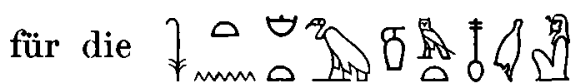

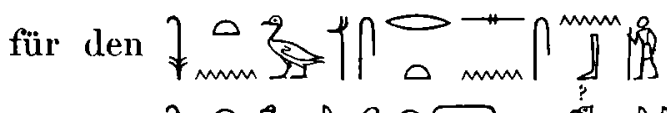

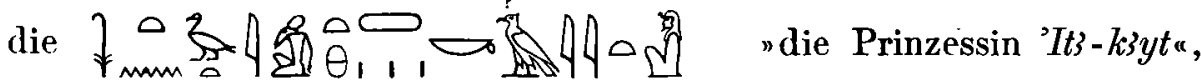

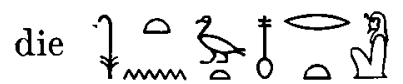

"die königliche Gemahlin und Mutter, die mit der weilsen Krone vereinigte ", "den Prinzen Wsrtsn-snb“, "die Prinzessin Nfrt" u.s.w.

Ihre Geburts- und Todestage scheinen im Tempel gefeiert zu werden. So finden wir z. B. auf verschiedenen Documenten die Fest des Zum-Himmel-aufsteigens “, womit nur der Todestag Usertesen's II. gemeint sein kann, auf den 14. des 4. Wintermonats angegeben.

Auch über das Todesjahr dieses Herrschers giebt eine Rechnung Auskunft. $\mathrm{Da}$ sie auch als Beispiel der Abfassung solcher Rechnungen interessant ist, so mag sie hier wiedergegeben werden:

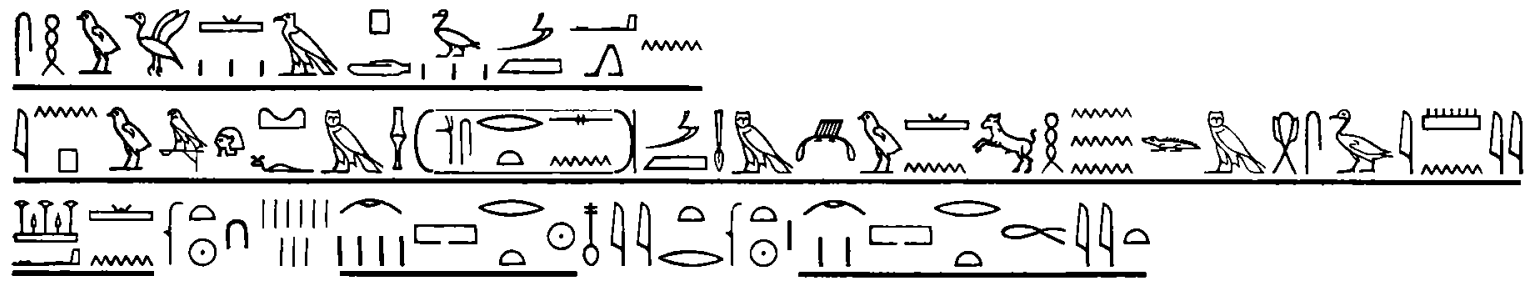




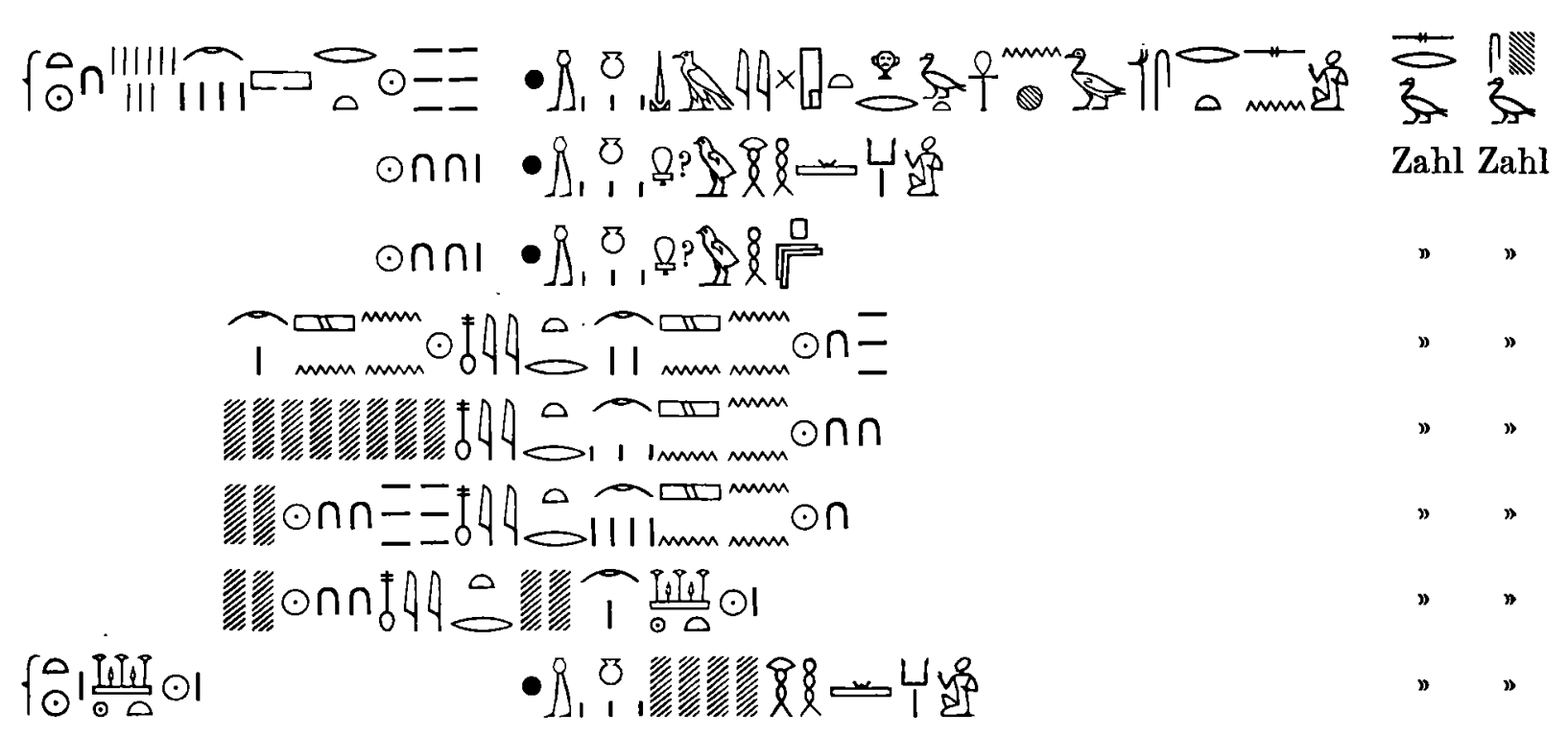

u. s. w.

"Liste der Gänse, die dem Anubis auf seinem Berge in "Mächtig ist der selige Usertesen " dargebracht wurden ....... der Sbk-m-s;s Sohn Imny. “

"Von Jahr 19, 4. Wintermonat, an bis Jahr 1, 2. Wintermonat, Ende."

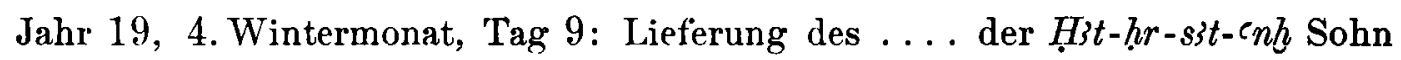
Wsrtsn, Sr-Gänse S-Gänse ...

Tag 21: Lieferung des ....W Wi $h-k 3$,

Tag 21: Lieferung des ....Hp,

1. Erntemonat bis 2., Tag 13,

... bis 3., Tag 20,

... Tag 26 bis 4., Tag 10 ,

... Tag 20 bis (Jahr 1) 1. Überschwemmungsmonat, Tag 1,

Jahr 1, 1. Überschwemmungsmonat, Tag 1: Lieferung des ....W3 $h-k \xi$

$$
\text { u. s. w. }
$$

Dieses Document lehrt uns: erstens, dafs Usertesen II. - denn die langen Regierungen seiner Nachfolger passen nicht zu unseren Angaben - 19 Jahre regiert hat, was zu dem im Turiner Papyrus enthaltenen Zahlenrest stimmt, und zweitens, dafs im m. R. die neue Zählung nicht vom Todestage des alten Königs, sondern erst von dem auf ihn folgenden ersten Thoth beginnt.

Über andere Fragen der altägyptischen Zeitbestimmung klärt uns die folgende Rechnung auf:

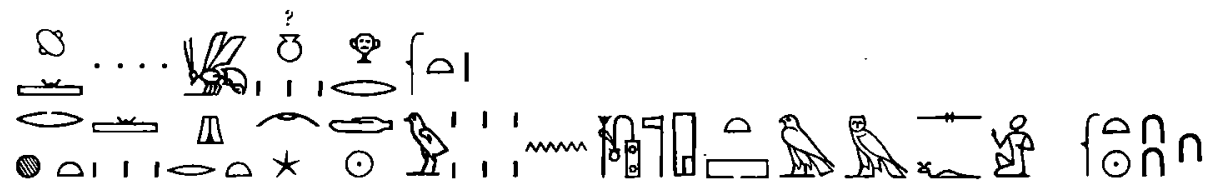

$$
\begin{aligned}
& \text { ש }
\end{aligned}
$$




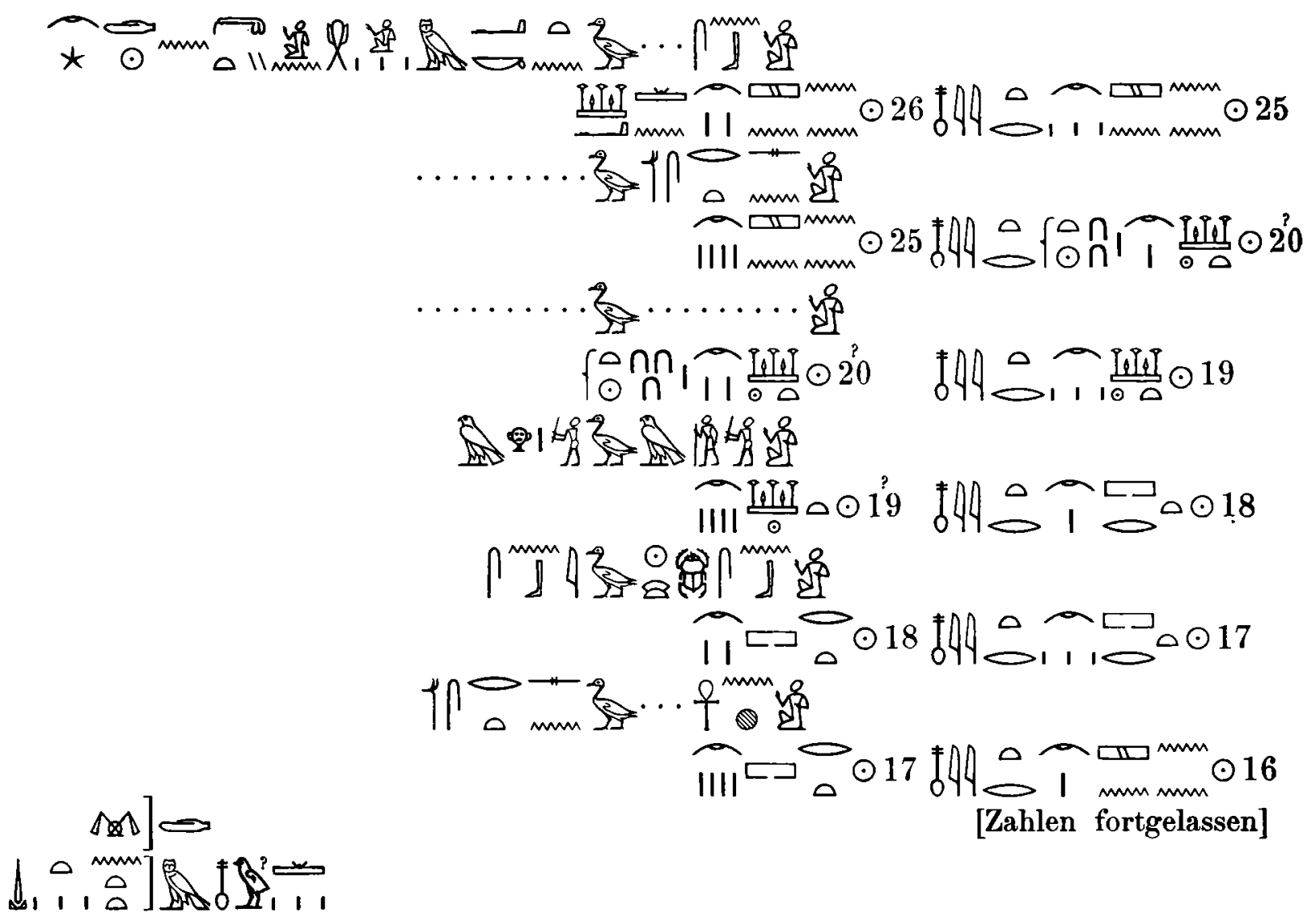

"Berechnung von .... und .... auf ein Jahr.

"Betrag der sechs Monatseinkünfte für den Tempelschreiber $\not r r-m-s\} f$. Jahr 31. "Abzug(?) von diesem Betrage:

"Monat des Abtheilungsvorstehers:

der Mcktn Sohn ....-snb, vom 26. d. 2. Erntem. bis 25. d. 3.

.... Sohn Wsrtsn, vom 25. d. 4. Erntem. bis 20. d. 1. Übersehw. Jahr 31.

.... Sohn ...., vom 20. d. 2. Überschw. bis 19. d. 3 .

des $H r-h r-n h t$ Sohn $H r-w r-n h t$, vom 19. d. 4. Überschw. bis 18. d.

1. Winterm.

des Snbi Sohn HC-hpr-Rc-snb, vom 18. d. 2. Winterm. bis 17. d. 3. des Wrtsn Sohn ....-cnh, vom 17. d. 4. Winterm. bis 16. d. 1. Erntem.

Summe:

Rest, der .....

[Zahlen fortgelassen]

Betrachten wir die hier gegebenen Daten genauer, so sehen wir, dals wir hier nur ein Jahr von 354 Tagen, also ein Mondjahr, vor uns haben und dafs die Grenzen der angegebenen Monate immer etwa 29 Tage, also einen Mondmonat, von einander entfernt sind. Die hier wohl gemeinten Neumonde sind aber, wie eine Rechnung ergiebt, nicht beobachtet, sondern nur durch Taxat 
bestimunt. Aber so viel geht sicher aus dieser Tabelle und aus anderen ähnlichen hervor, dafs die Einkünfte der Priester nach Mondmonaten berechnet wurden und dafs die Abtheilungsvorsteher im Jahre wechselten.

Über diesen Wechsel gewisser Priester — der uns als $x_{1}^{\circ}$ 号! "Laienpriesterschaft “ bekannten - giebt uns die folgende, einer Rechnung entnommene Liste willkommenes Material. Dieselbe zählt auf:

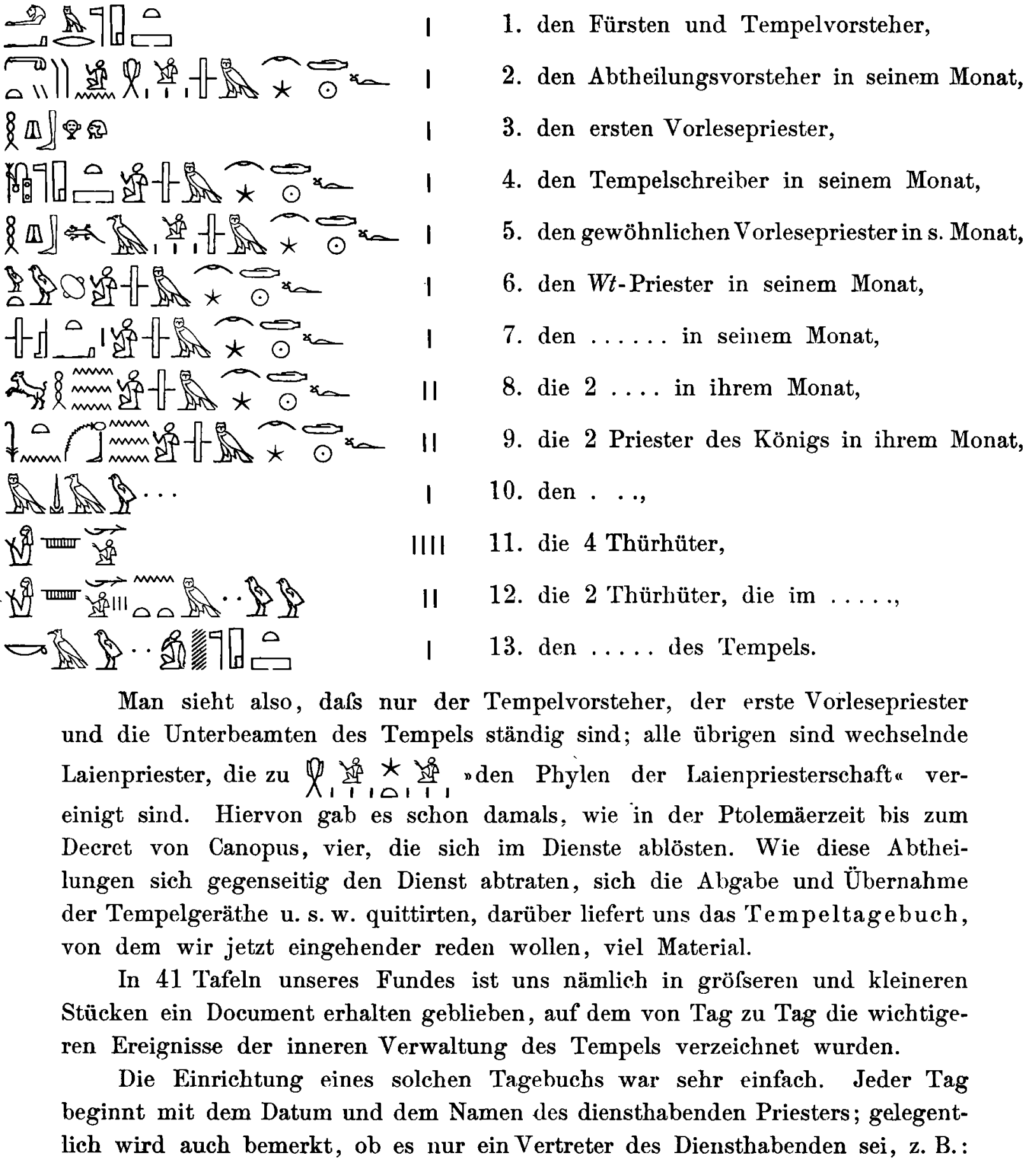




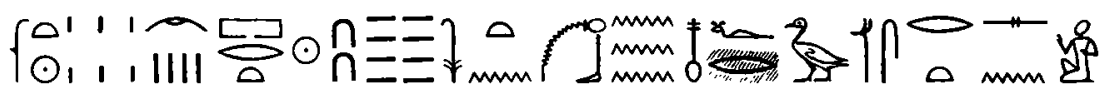

„Jahr 6, 4. Wintermonat, am 28. : der Priester des Königs, des Nfr Sohn, Wsrtsn. “

Dahinter folgen dann allerlei Notizen. Häufig sind Inventarverzeichnisse von Tempelgeräthen, in denen es etwa heifst:

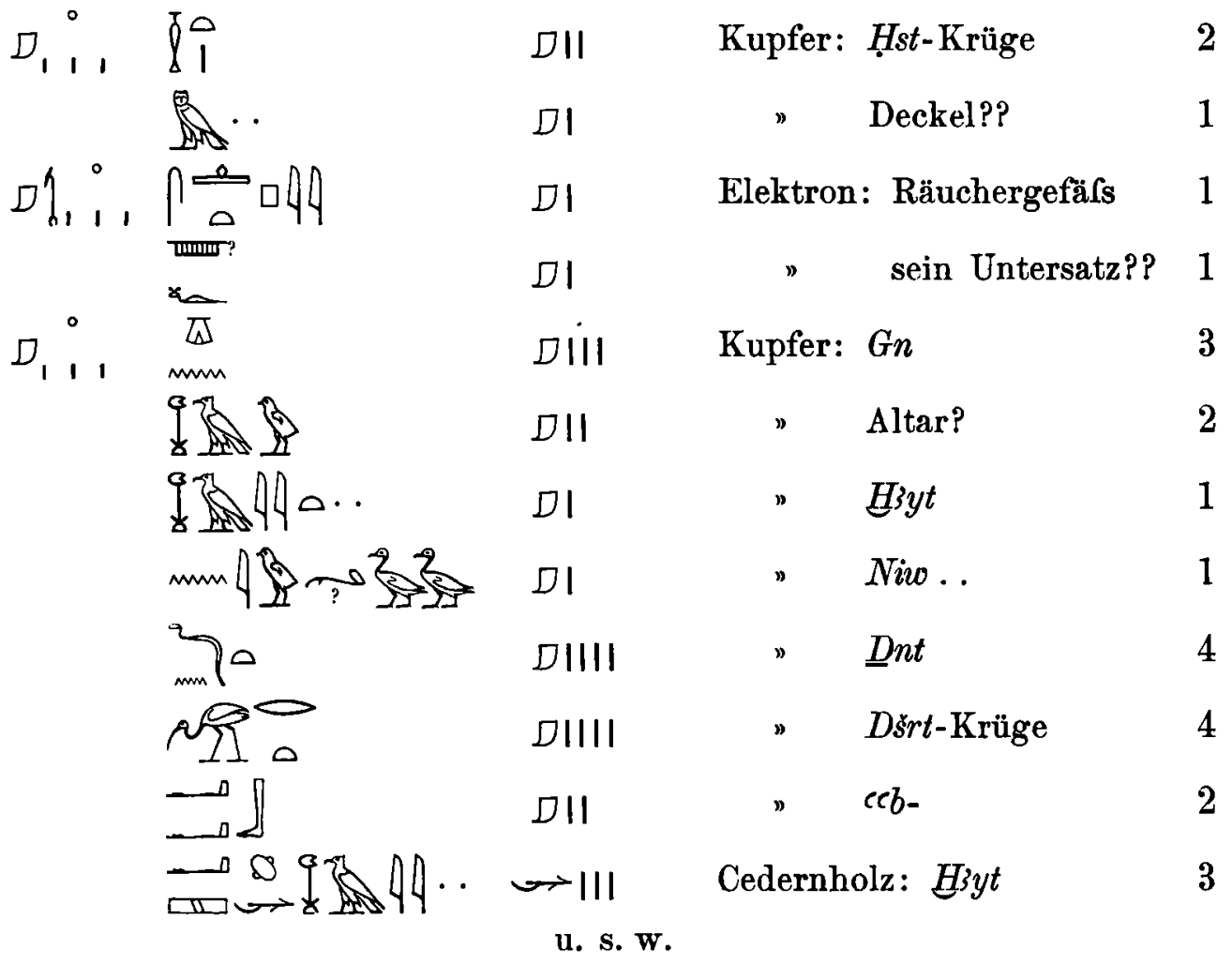

Interessanter wird solches Verzeichnifs, wenn gar Statuen inventarisirt werden, z. B. :

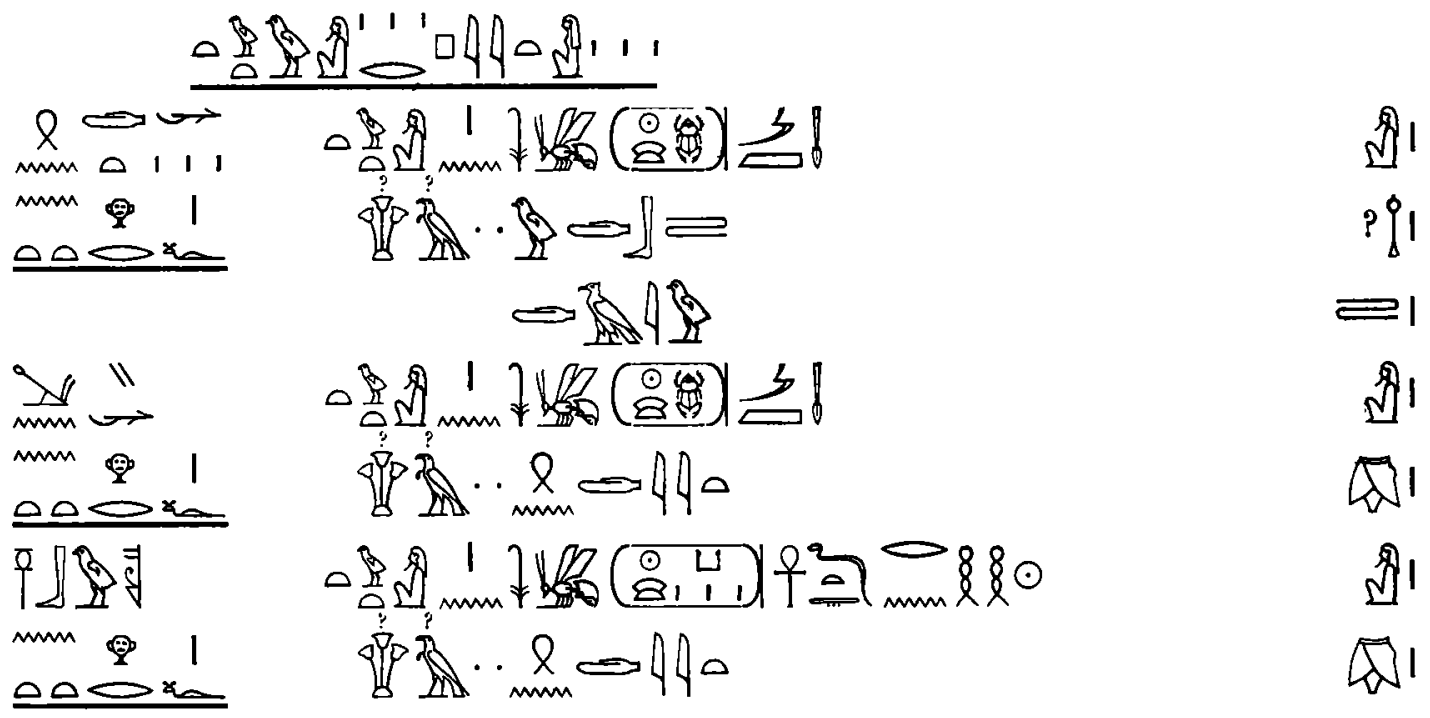




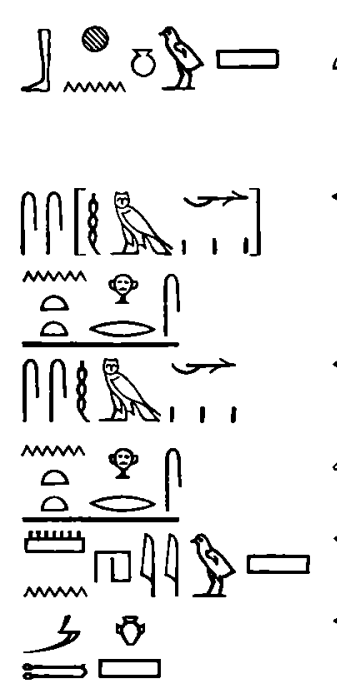

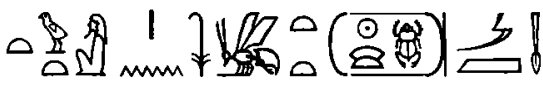

$$
\begin{aligned}
& (8,4) 7^{2} \underline{2} \text { 의 }
\end{aligned}
$$

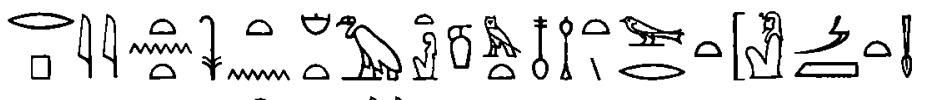$$
8=49 \circ
$$

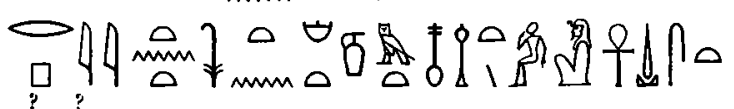$$
\text { is }=49 \circ
$$

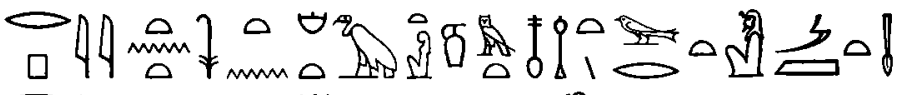

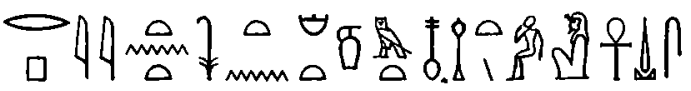

Statuen und Königinnenbilder

Akazienholz: Statue des seligen Us. II.

Was darauf ist: .... Hülle?

ถิ

กิ

螘

a

太ㄴ

Q

ฝी।

斻

.....

Ebenholz: Statue des seligen Us. II.

Was darauf ist: Schurz

Elfenbein: Statue des regierenden Us. III.

Was darauf ist: Schurz

Hammamat-Stein: Statue des seligen Us. II. 1

Statue des regierenden Us. III.

Ssnd $m$-Holz: Statue der königlichen Frau und Mutter, der mit der weilsen Krone vereinigten, Grofsen, der seligen

Was daran ist: Schurz

Ssndm-Holz: Statue der königlichen Frau, der mit der weilsen

Krone vereinigten, der Kleinen, der regierenden

Was daran ist: Schurz

Mnhyw-Stein: Statue der königlichen Frau und Mutter, der mit der weifsen Krone vereinigten, Grofsen, der seligen

Granit: Statue der königlichen Frau, der mit der weifsen Krone vereinigten, Kleinen, der regierenden

Es werden übrigens auch Statuen von Prinzen und Prinzessinnen, ja selbst solche von hohen Würdenträgern genannt, z. B.:

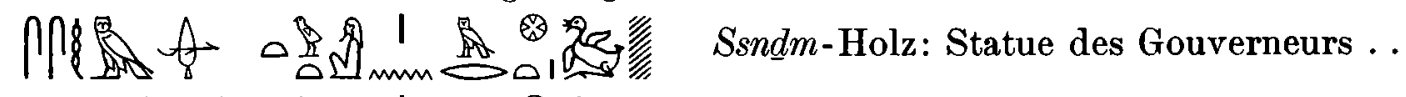

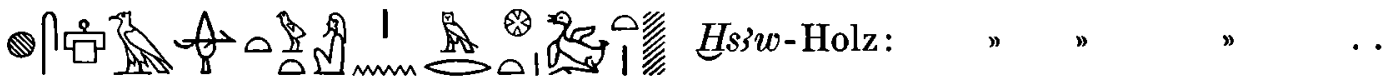

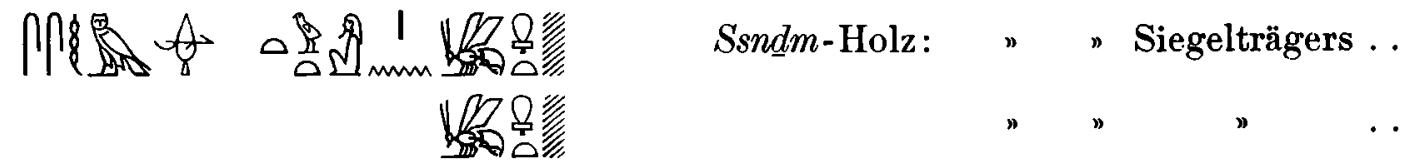

u. s. w. noch dreimal 


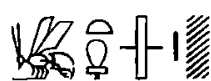

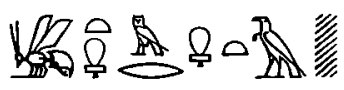

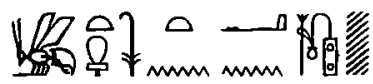

des Siegelträgers ..

" des Vorstehers der Schatzmeister des Schreibers vor dem Könige

Solche Inventarisirungen treten deshalb so häufig auf, weil sie sehr oft, anscheinend zum Zwecke der Übergabe des Tempelinventars von einer Phyle an die andere, aufgenommen werden mulsten. Auf die Inventare folgen nämlich ständig Abschriften von Übergabeverhandlungen, etwa in folgender Form:

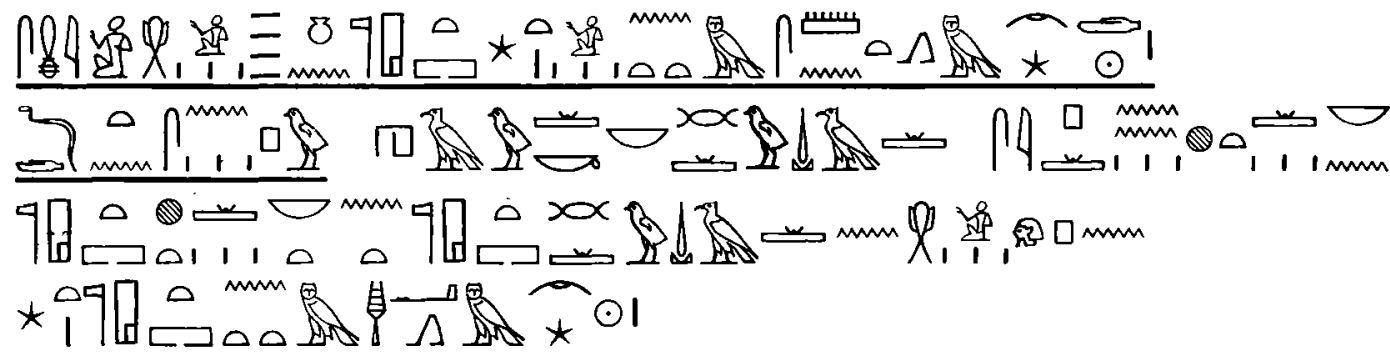

"Es berichtet die vierte Abtheilung des Tempels, die Laienpriesterschaft, die in diesem Monat abtritt.

Sie sagen nämlich: Alle deine .... sind in Ordnung. Wir haben alles Tempeleigenthum aufgenommen. Alles Tempeleigenthum ist in Ordnung für die erste Phyle der Laienpriester des Tempels, welche in diesem Monat antritt. “

Auf ein solches Abgabeattest folgt regelmälsig die Bescheinigung der Annahme:

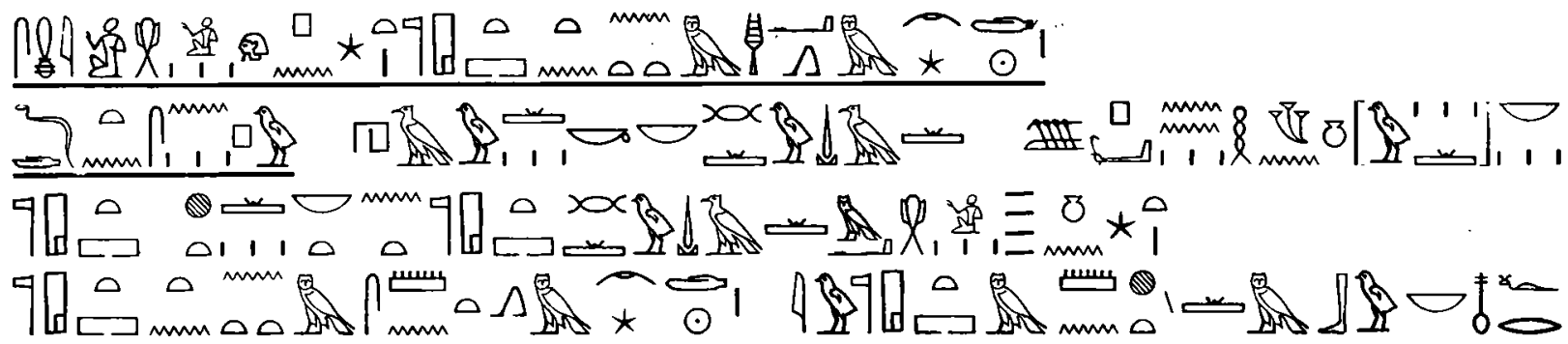

"Es berichtet die erste Abtheilung der Laienpriester dieses Tempels, die in diesem Monat antritt.

Sie sagen dies: Alle deine .... sind in Ordnung. Wir übernehmen alle Geräthe des Tempels, alles Eigenthum des Tempels in Ordnung von der vierten Phyle der Laienpriester dieses Tempels, die in diesem Monat abtritt. Der Tempel ist in gutem Zustande."

Diesen Verhandlungen pflegt dann die Namensliste der neu antretenden Phyle zu folgen:

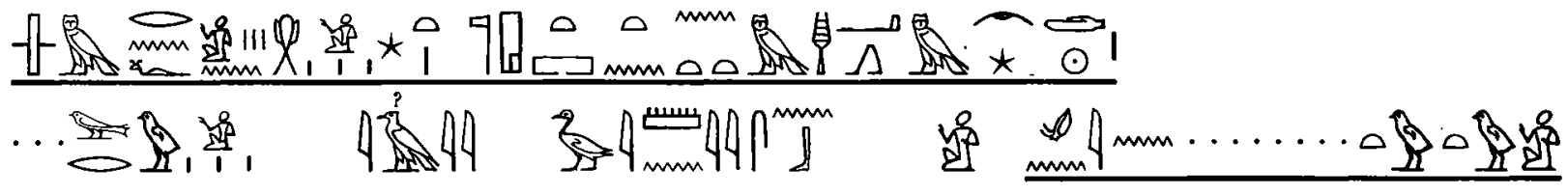




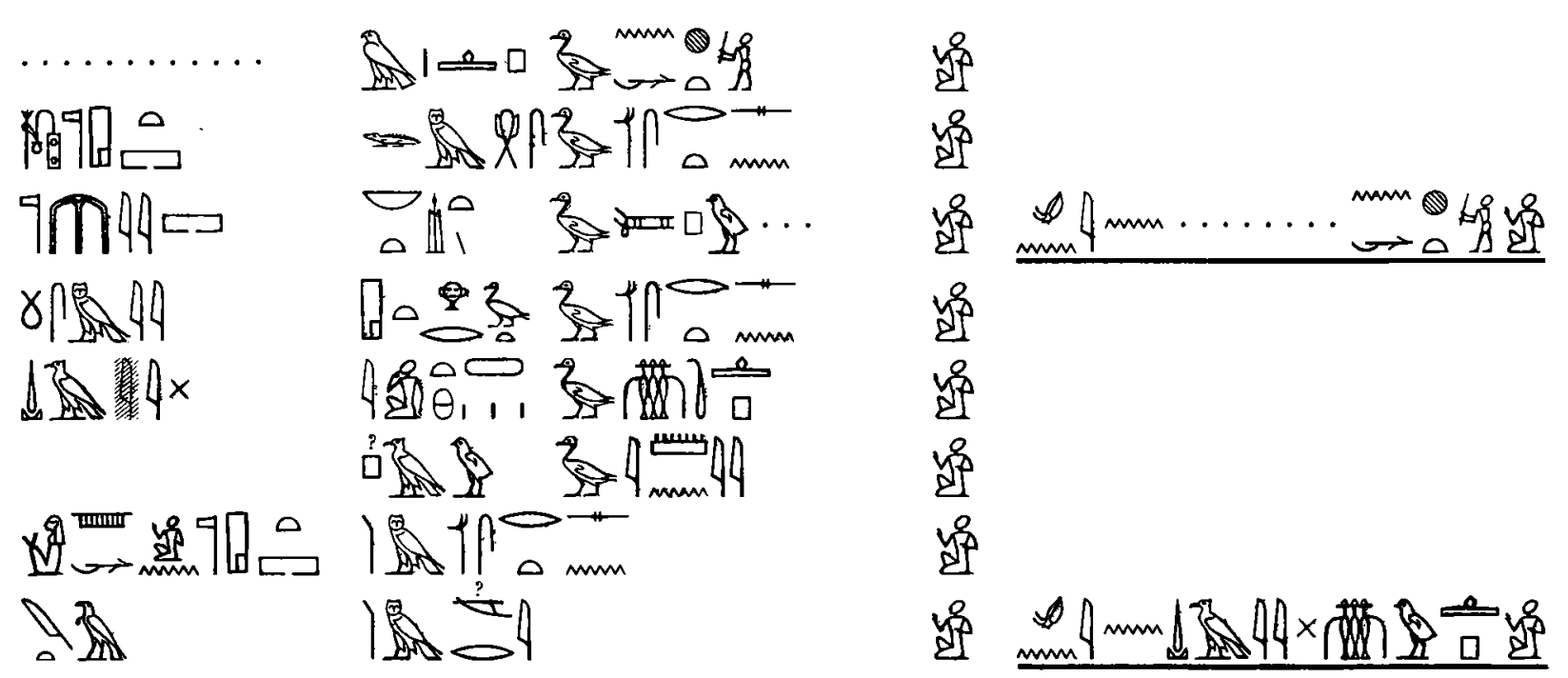

Namensliste der Laienpriesterabtheilung dieses Tempels, die in diesem Monat antritt:

.... Isp's Sohn 'Irnny-snb, vertreten durch ... . Twtw

.... Ḧr-htp's Sohn Nht.

Der Tempelschreiber $\$ b k-m-s\} s$ 's Sohn Wsrtsen

.... Nbt-Cnt's Sohn Tmw-pw..., vertreten durch ....Nht

$H t-\not h r-s 3 t$ 's Sohn Wsrtsn

.... Its's Sohn Hinti-htp

P3y's Sohn 'Fmnw.

Der 'Thürhüter, Asiat Wsrtsn

.... Asiat $M r i$, vertreten durch .... Hnti-htp.

Die übrigen Aufzeichnungen betreffen Tempeleinkünfte, Opferquittungen und Ähnliches. Es sind wohl meist A bschriften solcher Bestellzettel, wie wir Eingangs einen, den über das Rindsleder, erwähnten. Ein diesem gleicher Vermerk findet sich übrigens, wenn auch mit einem anderen Datum, in unserem Tagebuch; er lautet:

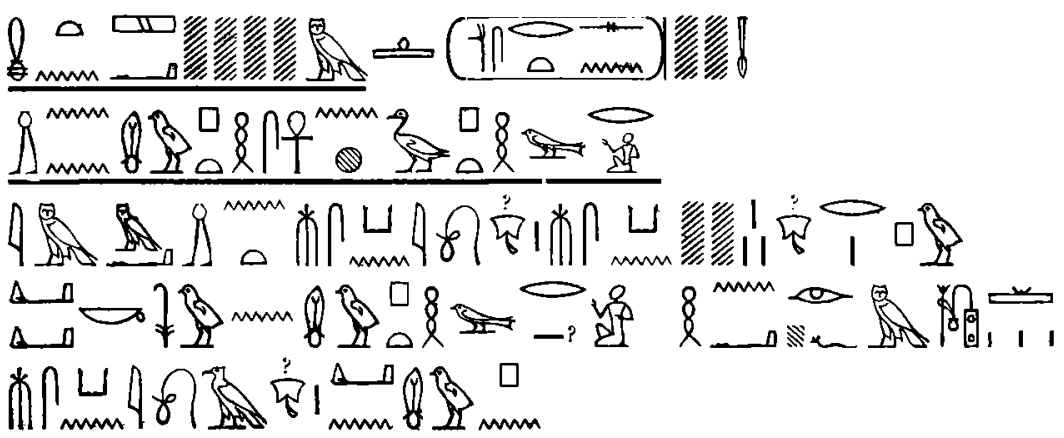

"Abschrift des Briefes .... nach der Stadt 'Zufrieden ist der selige Wsrtsn', nüberbracht von dem Schuster des Pth-s`nh Sohn, Pth-wr.

"Lals ein Rindsleder oder ein (. .?)-Leder bringen.

*Gieb es dem Schuster Pth-wr, und lasse es aufschreiben.

"1 Rindsleder diesem Schuster übergeben. * 
Das bei Weitem wichtigste Resultat, das die Durchsuchung dieses Tagebuchs bisher ergeben hat, ist aber ein chronologisches. Wir finden nämlich unter den Aufzeichnungen ein neues Sothisdatum, durch das unsere Kenntnisse der absoluten Chronologie der älteren ägyptischen Geschichte wieder um ein gutes Theil erweitert werden. Auf einem der Fragmente steht die folgende Notiz:

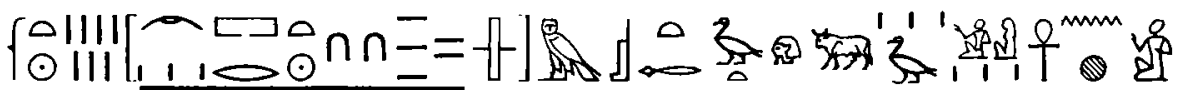

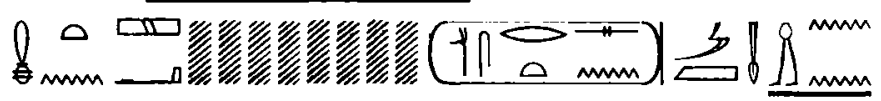

9.

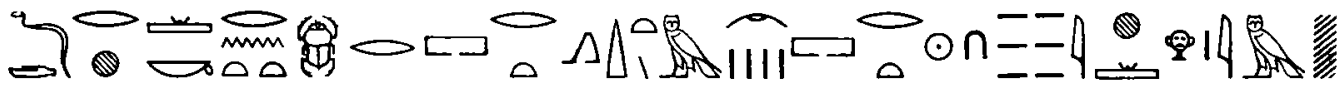

* 通,

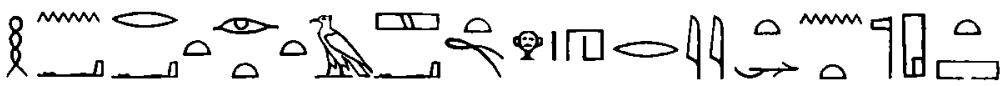

"Jahr 7 [3. Wintermonat, am 25. $\left.]^{1}\right) \ldots$ der $S 3 t-t p-i h w$ Sohn $R h w-c_{n h}$

"Abschrift des Briefes .... Stadt 'der selige Usertesen ist mächtig'

nüberbracht durch (nicht ausgefüllt)

"Der Fürst und Tempelvorsteher $N b-k \xi w-r e$ an den ersten Vorlesepriester Ppy-htp.

"Du sollst wissen, dafs der Aufgang des Sirius am 16. des 4. Wintermonats stattfindet. Mögest Du .... [benachrichtigen?] die Laienpriester des 'Tempels der Stadt 'mächtig ist der selige Usertesen' und des Anubis auf seinem Berge und des Suchos .... Und lasse diesen Brief an (das Anzeigebrett?) des Tempels machen. «

Zufälligerweise ist uns auch ein zu derselben Handschrift gehöriges Fragment erhalten, auf dem unter dem 17. des 4. Wintermonats des Jahres 7, also einen 'Tag nach jenem von dem Tempelvorsteher angekündigten Aufgange des Hundssterns, unter den Einkünften des Tempels vermerkt sind:

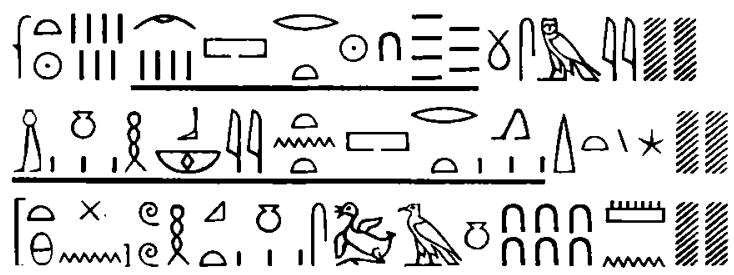

"Jahr 7, 4. Wintermonat, am 17. ...

"Einkünfte: Festgaben des Sothisaufganges ....

"200 verschiedene Brote. 60 Krüge Bier ....

Die zweite der eben genannten Notizen über den Siriusaufgang war übrigens bei der Durchmusterung des Fundes die erste, welche dem Verfasser in die Hände

1) Ergänzt nach den vorhergehenden Daten. 
fiel; an ihrer richtigen Lesung hat auch SETHE, der gerade anwesend war, wesentlichen Antheil.

Auf die Wichtigkeit, die beide Aufzeichnungen für die ägyptische Chronologie haben, brauchen Aegyptologen nicht erst besonders lingewiesen zu werden. Es ist allgemein bekannt, wie die bisher bekannten Angaben über Siriusaufgänge zur Fixirung verschiedener Punkte in der ägyptischen Geschichte gedient haben.

Das ägyptische Kalenderjahr, dessen Anfang theoretisch mit dem Frühaufgang des Hundssterns zusammenfallen sollte, bestand nur aus 12 Monaten zu je 30 Tagen und 5 zum letzten Monat hinzugerechneten Schalttagen, war also mit seinen 365 Tagen um rund $1 / 4$ Tag zu kurz. Die Folge davon war die Verschiebung des astronomischen Jahresanfanges, d.h. des Frülıaufganges des Hundssterns, gegen den kalendarischen Jahresanfang. Der Siriusaufgang fiel rund alle 4 Jahre um einen Tag weiter in das kalendarische Jahr hinein. Die Unzuträglichkeiten, die sich daraus ergaben, sind sehr anschaulich im Decret von Canopus geschildert worden, als es sich darum handelte, den Kalender zu reformiren:

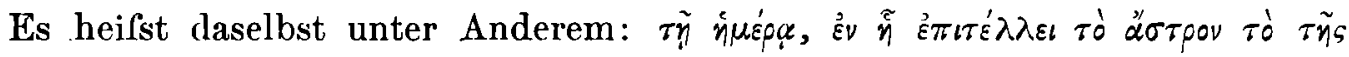

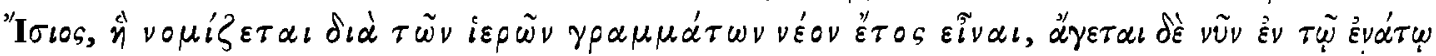

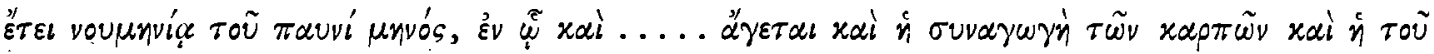

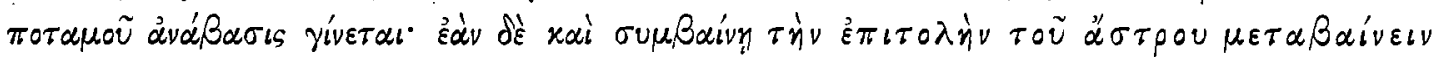

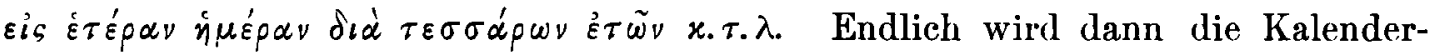
reform (die übrigens nur von kurzer Dauer gewesen ist) vorgeschlagen, "damit die Jahreszeiten wieder ihre Schuldigkeit thuen “.

Von einem früheren Versuch, den Kalender zu reformiren, wird hier nie gesprochen, trotzdem die Verfasser des Decrets sich stets auf die alten Schriften beziehen; es ist also wohl anzunehmen, dals bis auf die 'Tage Ptolemaeus' III. (Euergetes' I.) die Ägypter sich immer mit ihrem zu kurzen Jahre ohne vierjährige Schaltung beholfen haben. Es sind also alle vor dieser Zeit liegenden Sothisdaten für die absolute Fixirung der ägyptischen Chronologie verwendbar, und auch die nach Euergetes I. liegenden sind noch benutzbar, da seine Kalenderreform nur etwa 120 Jahre in Kraft war und dann, ohne eine Spur zu hinterlassen, verschwand. Die Ägypter müssen also neben dem Jahre des Euergetes, das wohl nur officiell in Gebrauch war, sich doch noch immer ihres alten Jahres bedient haben, wie sie ja auch später nach Einführung des augusteischen Jahres ruhig noch nach ihrem alten Kalender weiter rechnen, wie das die Doppeldatirungen nach dem "Jahre des Ioniers " und nach dem "Jahre des Ägypters" zeigen.

Die durch die sich regelmäfsig verschiebenden Sothisdaten ermittelten.Fixpunkte der ägyptischen Chronologie sind nun folgende:

1. der von Censorinus für das Jahr $139 \mathrm{n}$. Chr. angegebene Anfang einer Sothisperiode, wo also der Frühaufgang des Sirius am 1. des ersten Monats stattfand;

2. das Datum des Decrets von Canopus: Sothisaufgang am 1. des 10. Monats. 
Zwischen diesem und dem nächsten Datum fängt eine neue Sothisperiode an. Das von Petrie angenommene Sothisdatum aus der Regierung Merenptah's ist nicht als solches anzusehen, es folgt vielmehr erst:

3. die Kalendernotiz von Elephantine aus der Zeit Thutmosis' III.: Sothisaufgang am 28. des 11. Monats;

4. der EBers-Kalender aus dem 9. Jahre Amenophis' I.: Sothisaufgang am 9. des 11. Monats.

$\mathrm{Zu}$ diesen vier tritt nun als fünftes das aus unseren Papyri hinzu, nämlich das vom 7. Jahre Usertesen's III.: Sothisaufgang am 16. des 8. Monats.

Um allen Zweifeln zu begegnen, wollen wir erst erörtern, was uns zwingt, unsere Notiz in die Regierung Usertesen's III. zu setzen, trotzdem der Königsname an keiner der beiden Stellen ausdrücklich genannt ist. Die Fragmente des Tempeltagebuchs, aus dem unsere Aufzeichnungen entnommen sind, zeigen für die Jahre 5 bis 9 die gleiche Handschrift, eine kleine, sehr klare und deutliche, fette Schrift, die sich von den sonst auf unseren Papyri vorkommenden Schriften ganz charakteristisch unterscheidet. Es kann daher keinem Zweifel unterliegen, dafs in diesen fünf Jahren das Tempeltagebuch von ein und derselben Person geführt wurde. Nun sind aber in den Aufzeichnungen des neunten Jahres Königsnamen erwähnt, und zwar kommen mehrfach Statuen des "seligen “ Usertesen II. und des "ewig lebenden ", also regierenden, Usertesen III. vor. Hieraus folgt, dafs auch die von gleicher Hand geschriebenen Fragmente aus dem Jahre 7, die eben die Sothisdaten enthalten, unter Usertesen III. verfafst wurden.

Wir können also die Regierung Usertesen's III. nun astronomisch berechnen. Dies ist unter Zugrundelegung der von Oppozzer in den Sitzungsber. d. Kaiserl. Akad. d. Wiss. in Wien, Bd.90, Abth. II, S. 577 veröffentlichten Ermittelungen durch Hrn. Dr. Brix zu Berlin geschehen und ergiebt für das 7.Jahr Usertesen's III. die Jahre 1876 bis 1872 v. Chr.

Bei diesem Resultat müssen wir uns aber stets vergegenwärtigen, in welchen Fehlergrenzen es sich bewegen kann. Die Oppolzer'sche Formel selbst ergiebt einen möglichen Fehler von zwei Jahren; ferner berechnet Oppouzen nur die wirklichen Frühaufgänge des Sirius für die mittlere Breite von Mittelägypten. Nimmt man dagegen an, unser Datum vom 16. des 8. Monats bezöge sich auf einen irgendwie berechneten oder taxirten Siriusaufgang und auf irgend eine andere Breite, so wird sich das Resultat noch etwas, wenn auch nur wenig, verschieben. Endlich ist es auch unsicher, ob die Oppolzer'sche Annahme zu Recht besteht, dafs das Jahr 139 n. Chr. das erste Jahr der vierjährigen Periode des Zusammenfallens des astronomischen und kalendarischen Jahres der Ägypter sei. Brandis behauptet vielmehr auf Grund zweier Stellen des Ptolemaeus, dafs es das letzte Jahr eines solchen vierjährigen Zeitraums sei. Unter dieser Voraussetzung würde also unser Resultat sich um vier Jahre zurück verschieben. Wir sehen also, dals kleinere Ungewilsheiten bestehen bleiben, die 
aber bei der sonstigen Unschärfe der ägyptischen Chronologie zu unbedeutend sind, um weiter berücksichtigt zu werden. Selbst wenn man durchaus anzweifeln wollte, dafs das Datum sich auf Usertesen III. bezöge und etwa Usertesen II. oder Amenemhêt III., die beiden einzigen nocl möglichen Könige, dafür einsetzen wollte, würde man nur eine Verschiebung von 19 bez. 38 Jahren erhalten, was für diese weit zurückliegenden Zeiten auch noch nicht so unerhört wäre.

Es ist also clas 7. Jahr Usertesen's III. als in die Jahre von 1876-1873 v. Chr. fallend anzusehen, d. h. immer noch etwa 100 Jahre später als es der am niedrigsten greifende Historiker Ägyptens, Edvard Meyer, in seinen Minimaldaten annahm. Es bleiben uns also für die Zeit zwischen Usertesen III. und Amenophis I., dessen 9. Jahr durch die Sothisangabe des Papyrus Erers auf die Jahre von $1545-1542$ v. Chr. bestimmt ist, nur rund 330 Jahre und für die Zeit vom Ende der 12. bis zum Anfang der 18. Dynastie gar nur 200 bis 210 Jahre ülorig. Ist das mit den sonst bekannten historischen Angaben vereinbar?

Zuerst scheinen die in der Überlieferung nach Manetho gegebenen Zahlen dagegen zu sprechen. Aber diese sind so ungereimt, dafs sie bisher überhaupt kein Bearbeiter der ägyptischen Chronologie hat stehen lassen. Er giebt für die fragliche Periode der 13. bis einschliefslich 17. Dynastie nämlich zusammen 1350 Jahre an.

Dann scheint der Turiner Papyrus mit seiner grolsen Anzahl von Königsnamen unserer Annahme zu widersprechen. Diese Königsliste, die übrigens in ihren Angaben, wie wir oben gesehen haben, durch unseren neuen Fund sehr schön bestätigt wird, giebt aber hier nur Namen; die Zahlen sind leider zerstört, sonst würden wir wohl gesehen haben, dafs alle diese Herrscher der 13. und 14. Dynastie nur ganz ephemere waren. Die anderen Königslisten überspringen die fragliche Epoche fast gänzlich, woraus auch wiederum zu schliefsen, dafs sie wohl nur wenig Zeit ausfïllte. Endlich ist der Mangel an Denkmälern aus jener Zwischenzeit sehr grols, was wohl auch nur auf das Fehlen langer Regierungslauern zurïckzuführen ist.

Dies Alles sind Gesichtspunkte, die die Annahme von nur zwei Jahrhunderten als Zwischenzeit zwischen 12. und 18. Dynastie wahrscheinlich machen. Ferner mufs aber die Betraclitung der Kunstentwickelung uns davon überzeugen, dafs man bisher jene Epoche viel zu lang annahm. Henry Wallis wies bereits früher darauf hin, dafs die geringe Weiterentwickelung der Kunst zwischen mittlerem und neuem Reich eigentlich verbiete, einen grofsen zeitlichen Abstand zwischen beiden Epochen zu construiren; und wenn man berücksichtigt, dafs wirklich schon ein archaeologisch sehr geschulter Blick dazu gehört, um eine Sculptur aus den ersten Jahren 'Thutmosis' III. von einer aus dem Ende der 12. Dynastie zu unterscheiden, so wird man dieser W beitreten können. Also auch in Hinblick auf die Kunstgeschichte dürfen wir mit der neuen Ansetzung des mittleren Reiches zufrieden sein. 
Neben der engeren ägyptischen Kunstgeschichte wird aber auch die weitere allgemeine Culturgeschichte von unserer neuen Feststellung Nutzen ziehen können. Sollten sich nämlich die von PETrre bei Illahun gefundenen Scherben nicht ägyptischer Herkunft wirklich, wie es den Anschein hat, als der ältesten mykenischen Periode entstammend erweisen, so wäre durch unsere neue Datirung der 12. Dynastie auch diese erste mykenische Periode in das 19. Jahrhundert v. Chr. gewiesen, eine Zeit, die, wie Prof. Loescrke mittheilt, ihm auch aus anderen Gründen sehr wahrscheinlich ist.

\title{
Ein Pyramidentext in ursprünglicher Fassung.
}

\author{
Von Fr. W. v. Bissing und L. Borchardt.
}

\begin{abstract}
Als im Winter 1897/98 Hr. Dr. Heñze, damals dem Kaiserl. Deutschen Generalconsulate zu Kairo attachirt, für das Altägyptische Wörterbuch die beschriebenen Pyramiden bei Saqqarah wieder öffinen und ihre Inschriften vollständig abklatschen liefs, machte in der Pyramide Pepi's I. einer der Arbeiter den zweiten Verfasser dieses Aufsatzes auf die vielen alten Correcturen ${ }^{1}$ ) aufmerksam, die sich dort in den Inschriften des südlichen, hinter den Fallsteinen gelegenen Theiles des Einganges [Z. 234-389 der Maspero'schen Publication] finden.

Da man sofort sehen konnte, dafs diese Textveränderungen sich fast nur auf den Namen des Verstorbenen sowie auf die Personalpronomina und -suffixe beschränkten, also durch die Umsetzung einer anderen, älteren Fassung des ganzen Textes in die jetzt vorhandene bedingt waren, da auch in der Veröffentlichung diese Correcturen nur gelegentlich erwähnt werden, so lohnte es sich wohl der Mühe, den ganzen corrigirten Theil mit besonderer Berücksichtigung der veränderten Stellen noch einmal genau durchzusehen.

Hierbei wurde so vorgegangen: zuerst wurden von beiden Verfassern gemeinsam in die Maspero'sche Publication die unter dem heutigen Text noch

1) Diese sind entweder durch Ausmeifselung oder so hergestellt, dafs die alte Lesart nur mit Gipsmörtel verschmiert und dann die neue darübergeschnitten ist. Der ausgefallene Gips lälst jetzt vielfach beide Lesungen erkennen; an den Stellen, wo er noch haftet, zeigten sie die verschiedene Färbung oder kleine Niveau-Unterschiede an.
\end{abstract}

